\title{
Envejecimiento y mortalidad: condiciones de salud, económicas y familiares en el último año de vida de las personas mayores en México
}

\section{Aging and mortality: health, economic and family conditions in the last year of life of the elderly in Mexico}

\author{
César Alfredo González-González* \\ Karina Orozco-Rocha** \\ Mireya Patricia Arias-Soto* \\ María Gregoria Carbajal-Santillán* \\ Rafael Samper-Ternent*** \\ * Facultad de Trabajo Social de la Universidad de Colima, Colima, México \\ ** Consultora privada \\ *** University of Texas Medical Branch, Galveston, Texas, Estados Unidos
}

\section{Resumen}

Poco se sabe sobre lo que sucede en el último año de vida de la población envejecida. México presenta características demográficas, económicas, sociales y epidemiológicas que plantean inquietudes sobre los desafíos que el envejecimiento impondrá. El objetivo es analizar el último año de vida de la población mayor mexicana y describir el estado de salud, el funcionamiento físico, el uso de los servicios de salud y el apoyo familiar que tenían antes de morir. Se utiliza información del Estudio Nacional de Salud y Envejecimiento en México (ENASEM). Los resultados señalan que durante el último año de vida, la presencia de enfermedades crónicas, dificultad en las actividades de la vida diaria, apoyo por parte de la familia y el uso de servicios de salud son una constante tanto para el individuo como para la familia.

Palabras clave: Envejecimiento, mortalidad, hospitalización, enfermedades crónicas, arreglos residenciales.

\section{Abstract}

Little is known on what happens in the last year of life of the aging population. Mexico has demographic, economic, social and epidemiological characteristics that raise concerns about the challenges that aging will impose. The objective is to analyze the last year of life of the Mexican elderly population and describe the state of health, physical functioning, use of health services and family support that they had before death. We used information from the Mexican Health and Aging Study (MHAS). The results indicate that during the last year of life, the presence of chronic diseases, difficulty in activities of daily living, support from the family and the use of health services are a constant for both the individual and the family.

Keywords: Aging, mortality, hospitalization, chronic diseases, living arrangements. 


\section{INTRODUCCIÓN}

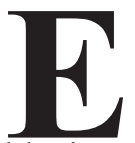

1 estudio de la mortalidad en las edades avanzadas ha sido ampliamente abordado, pero poco se conoce de lo que pasa en el último año de vida. Es escasa la evidencia empírica a nivel poblacional que señale las condiciones económicas, familiares y de salud durante el último año de vida de la población en edades avanzadas. La mayoría de los análisis sobre mortalidad han sido abordados con datos transversales (Monteverde et al., 2010), analizando los factores asociados con la mortalidad (Prince et al., 2012; González-González et al., 2014; González-González, Palloni y Wong, 2015), los factores predictores de la mortalidad (García-González et al., 2009; Schonberg et al., 2009), pero pocos se han enfocado a describir las características y condiciones en esta parte final de la vida, en especial en el último año de vida.

Las investigaciones sobre la mortalidad y lo que sucede en el último año de vida por lo general se han realizado en países desarrollados (Lunney et al., 2003; Boyd et al., 2008; Hwang et al., 2016), pero no se sabe si las conclusiones obtenidas de esos estudios son válidas para un país como México. Por ejemplo, se sabe que la mortalidad está asociada con largos periodos con enfermedades crónicas, discapacidad prolongada, alto número de visitas médicas en instituciones de cuidados a la salud, y cambios en los arreglos residenciales, pero en México no se ha investigado si estas condiciones se intensifican en el último año de vida.

Este periodo, el último año de vida, es de particular importancia para avanzar en el conocimiento sobre el proceso morbilidad-mortalidad de las personas mayores. Se ha documentado que el último año de vida es de particular importancia por ser un periodo en el que se concentran dificultades tanto para el adulto mayor como para las familias, particularmente en la provisión de servicios de salud. Por ejemplo, en el último año de vida se presentan cambios importantes, tanto en el estado físico, como en el cognitivo que lleva al declive funcional y después a la muerte (Lunney et al., 2003; Boyd et al., 2008). Estos cambios representan un reto mayor para el individuo, la familia, los cuidadores y los profesionales de la salud que proveen cuidado al final de la vida, y también es un factor importante en la utilización de distintos servicios de salud y el gasto asociado a los mismos.

Payne, Laporte, Deber y Coyte (2007) señalan que no es la muerte la que incrementa los gastos sino la morbilidad que precede y eventualmente 
lleva a la muerte. Estudios sobre el sistema de atención Medicare en Estados Unidos han demostrado que en las últimas dos décadas, alrededor de un cuarto de la provisión de sus servicios son para el último año de vida (Hogan et al., 2001). También se ha encontrado que los gastos de aquellos beneficiarios de Medicare y que murieron fueron seis veces mayores que los gastos registrados para los sobrevivientes (Lubitz y Riley, 1993).

México presenta una oportunidad única para analizar las condiciones familiares, económicas y de salud durante el último año de vida de la población en edades avanzadas por varias razones. Primero, estamos en medio de un proceso de envejecimiento poblacional acelerado que se intensificará en las siguientes décadas (CONAPO, 2014). Segundo, México es un país heterogéneo y con amplias desigualdades sociales y económicas que permiten analizar las diferencias en mortalidad por estrato socioeconómico (Muradás Troitiño, 2010). Tercero, producto de la transición epidemiológica que aún no concluye, en México se presenta un régimen epidemiológico mixto, en donde las enfermedades crónicas son las principales causas de morbilidad y mortalidad pero con las enfermedades infecciosas aún con un papel importante y que combinadas (crónicas e infecciosas) exacerban el efecto en la mortalidad (González-González, Palloni y Wong, 2015). Y cuarto, la población en edades avanzadas vive con largos periodos de discapacidad y que se agravan al alcanzar los 85 años o más, lo cual impacta de manera diferenciada en los recursos que se asignan y en la provisión de cuidados que se requieren (Marín y Casanovas, 2001). La conjugación de estos elementos hace suponer que el envejecimiento poblacional impondrá grandes retos a los sistemas sociales, económicos y de salud.

Por lo tanto, el objetivo principal de este artículo es describir el último año de vida de las personas adultas mayores mexicanos en relación con el estado de salud, la funcionalidad física, así como los arreglos y apoyos familiares. Se trata de describir cada una de estas dimensiones por separado, analizar cómo se modifican o complementan, y bajo qué circunstancias se está presentando en las personas adultas mayores en México.

\section{Metodología}

Para llevar a cabo esta investigación se utilizó información del Estudio Nacional sobre Salud y Envejecimiento en México (ENASEM). Es el primer estudio en México con información longitudinal, enfocado a la población en edades avanzadas, es decir, aquellos que tienen 50 años o más. El ENASEM consta de cuatro rondas de información, la basal levantada en 2001 y tres de seguimiento, una en 2003, otra en 2012 y la más reciente llevada 
a cabo en 2015; lo que significa que para algunos sujetos entrevistados se tiene información recogida durante 14 años de seguimiento.

En el año 2001 se entrevistó a 15,402 individuos, en 2012 para mantener la representatividad de la población de 50 años o más se agregó una muestra nueva de población entre 50 y 61 años. En lo que a mortalidad se refiere, entre 2001 y 2003 hubo 542 muertes y entre 2003 y 2012 un total de 2,742 participantes fallecieron, lo que da un acumulado de 3,284 muertes. En este artículo nos vamos a centrar en los fallecidos entre la ronda dos $\mathrm{y}$ tres $(\mathrm{n}=2,742)$ una muestra suficiente para analizar lo que sucede en el último año de vida de la población de personas mayores en México.

Sí un participante del estudio fallece, se aplica una entrevista por fallecimiento, la cual es obtenida a través de un proxy o sustituto, es decir, la información es proporcionada por un informante clave que por lo general fue el cuidador principal del sujeto de estudio. La entrevista de salida incluye información sobre datos demográficos (sexo, edad, escolaridad, estado civil, historia de migración, etc.), salud (auto-reporte de siete enfermedades crónicas, peso y talla, consumo de tabaco, consumo de alcohol), capacidad funcional (dificultad para realizar las actividades básicas e instrumentales de la vida diaria), no poder estar solo (relacionado con deterioro cognitivo y demencias), empleo y bienes (historia laboral, participación económica actual, negocios, pensiones, etc.) y por lo general se refiere a las condiciones de vida durante el último año del fallecido. Además, para cada una de las personas fallecidas se puede tener la información de rondas previas.

Con la finalidad de resaltar la heterogeneidad que se presenta en las condiciones de cada individuo en el periodo previo a su muerte, específicamente durante el último año de vida de los personas mayores, el análisis se hará con las características reportadas en 2001 y diferenciando por grupos de edad (50-59, 60-69, 70-79, 80-89, 90 o años), sexo y tamaño de localidad (1-2499, 2500-14999, 15000-99999, 100000 o habitantes).

La estrategia de análisis se basa en una descripción detallada de las características demográficas, de las condiciones de salud y del uso de servicios de salud durante el último año de vida de la persona mayor; dado que este es un artículo con un enfoque mayormente descriptivo, la pertinencia conceptual y la relación de la mortalidad con las características demográficas de los fallecidos se aborda en la sección de resultados. Para complementar el análisis se elaboró un modelo de regresión para analizar los factores asociados con los días de hospitalización para profundizar en el uso de servicios de salud (un proxy de las dificultades que el adulto mayor y su familia enfrentaron durante el último año de vida). 


\section{Resultados}

En el Estudio Nacional sobre Salud y Envejecimiento en México (ENASEM), en 2012, se obtuvo información de 10,989 personas que en 2003 habían sido entrevistados. Para 2012 se reportan 2,638 fallecidos, lo que representa 24.9 por ciento de la población total, la mortalidad es más alta entre los hombres pues 27.8 por ciento falleció en este periodo, lo mismo que 22.5 por ciento de las mujeres. El promedio de edad al que fallecieron las personas adultas mayores fue 77.4 años.

El lugar en dónde se muere (casa, institución u otro) se relaciona con los cuidados o complicaciones que las personas adultas mayores pudieron tener antes de morir. Los informantes clave reportan que 61.7 por ciento de los fallecidos murieron en su casa, 35.9 por ciento en un hospital o institución y 2.4 por ciento en otro lugar. En los resultados se observan diferencias con respecto al lugar en que se falleció, por grupos de edad, de los que tenían entre 50 y 59 años un 49.7 por ciento falleció en su casa mientras que para el grupo de 90 años o más el porcentaje fue 78.8. También se encontraron diferencias por el tamaño de la localidad de residencia, los que vivían en localidades más urbanas (100 mil o más habitantes), poco menos de la mitad (46.2 por ciento) falleció en casa, lo mismo que 76.2 por ciento de los que vivían en localidades rurales (menos de 2,500 habitantes).

Los informantes sustitutos reportaron que para 83 por ciento de los fallecidos la principal causa de muerte fue una enfermedad, en 5.8 por ciento de los casos fue violencia o accidentes y en 11.2 por ciento fue otra causa. Los resultados descriptivos señalan que no hay diferencias significativas por sexo en cuanto a la causa de muerte, lo que sí es evidente es la importancia de los accidentes o violencia como causa de muerte entre la población de 50-59 años, pues un 29.2 por ciento murió por esa causa mientras que en los otros grupos de edad el porcentaje es menor al seis por ciento.

\section{Estado de salud}

Entre los retos que genera el proceso de envejecimiento, la salud de la población en edades avanzadas es uno de los que más preocupa. Inherente al proceso de envejecimiento individual está la pérdida de la reserva funcional, según Alonso y colaboradores (2007) ésta pérdida condiciona una mayor susceptibilidad a la agresión externa al disminuir los mecanismos de respuesta y su eficacia para conservar el equilibrio interno, lo que puede desencadenar pérdida de la función, discapacidad y dependencia. 
Las enfermedades crónicas en la población de adultos mayores representan un tema muy importante. De acuerdo con Cordova-Villalobos et al. (2008: 420), lo son por "el gran número de casos afectados, su creciente contribución a la mortalidad general, la conformación en la causa más frecuente de incapacidad prematura y la complejidad y costo elevado de su tratamiento".

Los resultados del ENASEM señalan que entre los que fallecieron por una enfermedad, ataque al corazón fue la causa más frecuente con 19.6 por ciento de los casos, seguido por cáncer con 18.8 por ciento, diabetes con 17 por ciento y en 38.3 por ciento de los casos otra enfermedad causó la muerte. Los datos del ENASEM señalan que en los hombres el ataque al corazón (24.2 por ciento) fue la principal enfermedad que causó la muerte, mientras que en las mujeres fue la diabetes (22.3 por ciento). Por grupos de edad, destacan el cáncer y la diabetes pues dan cuenta de casi la mitad de las muertes por enfermedad en el grupo de 50-59 y 60-69 años, mientras que en el grupo de 90 años o más son otras enfermedades ${ }^{1}$ las principales causas de muerte, esto deja ver una gama más amplia de enfermedades que causan la muerte en las edades muy avanzadas. Por tamaño de localidad, en las localidades más urbanas se reporta que en 20.3 por ciento de los casos la diabetes fue la principal causa de muerte, casi el doble de lo que representa en las localidades rurales (13.4 por ciento).

En cuanto a las enfermedades crónicas que los fallecidos tenían diagnosticadas antes de morir (no la principal causa de muerte), a 30.7 por ciento un médico les había dicho que tenían diabetes, a 18 por ciento de los fallecidos les habían diagnosticado con cáncer o un tumor maligno, 12.7 por ciento tenían una enfermedad respiratoria, 16.1 por ciento había tenido un ataque al corazón y 9.4 por ciento tuvo una embolia.

Específicamente con la diabetes, destaca la diferencia en el porcentaje entre hombres y mujeres que tenían diagnosticada la enfermedad, 24.9 y 36.9 por ciento, respectivamente. También se reportan diferencias por tamaño de localidad de residencia, mientras que en los ámbitos más urbanos 34.6 por ciento de la población fallecida tenía diagnosticada diabetes, el porcentaje entre los que vivían en comunidades rurales era de 21.1 por ciento, diferencia que se puede explicar por los estilos de vida prevalecientes en cada entorno (Mohan et al., 2008).

Además de las enfermedades crónicas, el auto-reporte o la auto-percepción del estado de salud es uno de los indicadores más utilizados para me-

\footnotetext{
${ }^{1}$ En ENASEM se pregunta por otras enfermedades que causaron la muerte, pero se pregunta cómo una categoría, no hay una desagregación de esas enfermedades.
} 
dir el estado de salud de la población. Si bien es cierto que algunos autores señalan que la autopercepción que los individuos tienen sobre su estado de salud puede estar sesgada por problemas de endogeneidad y errores de medida (R. Todd Jewell, Rossi, 2007) y que estos errores de percepción son mayores cuando se le pregunta a un informante sustituto, también es cierto que en la mayoría de las investigaciones el auto-reporte de salud ha probado ser un indicador confiable para medir el estado de salud de la población y presenta ventajas por la facilidad con que se puede aplicar y responder (Frankenberg y Jones, 2004) y por eso se incluye en esta investigación.

Según lo reportado por los informantes sustitutos, de la población en edades avanzadas que falleció entre 2003 y 2012, 68.2 por ciento tenían un estado de salud regular o malo, es decir, dos de cada tres tenían un estado de salud deteriorado durante el último año de su vida. En comparación con los hombres (64.3 por ciento), un porcentaje mayor de mujeres tenían peor estado de salud (72.3 por ciento). Es importante mencionar que se encontró que entre los fallecidos del grupo de 90 años o más, 46 por ciento de los cuidadores reportó que las personas mayores tenían un buen estado de salud.

Las caídas son motivo de preocupación por la discapacidad que provocan, son un elemento importante en la salud del adulto mayor y están relacionadas con la mortalidad (Coutinho, Bloch y Coeli, 2012). Los resultados señalan que 39.1 por ciento de los fallecidos tuvo una caída durante el último año de vida y que 11.7 por ciento sufrió una fractura. En porcentaje, las mujeres sufrieron más caídas ( 42.6 por ciento) que los hombres $(35.8$ por ciento) y por lo tanto un porcentaje mayor también presentó fracturas.

Como se puede apreciar en las estadísticas descriptivas, la población de personas mayores presenta un estado de salud deteriorado y con diferencias importantes según las características de los individuos.

\section{Apoyo familiar}

Otro de los elementos importantes a analizar durante el último año de vida de las personas mayores es el apoyo familiar. En este sentido, la configuración de los arreglos residenciales tiene gran relevancia, es uno de los aspectos estrechamente relacionados con el bienestar en las edades avanzadas, así como su integración a distintos grupos de pertenencia (MacíasVega, 2004). El hogar es una de las fuentes principales de intercambios afectivos, de apoyo material y económico, por lo que tener a algún familiar cerca impacta directamente en la salud y calidad de vida de las personas mayores. 
Uno de los factores que por lo general reconfiguran las formas de convivencia familiar, son las enfermedades crónicas, pues generan discapacidad, dificultan la realización de las actividades cotidianas y esto obliga a que se incorporen nuevos miembros al hogar de la persona mayor enferma o que la persona mayor sea quien se cambie de hogar o vivienda para que le provean cuidados. En la ENASEM hay dos preguntas sobre los arreglos residenciales de las personas mayores durante el último año de su vida. La primera indaga sobre sí "En el último año de su vida, ¿se cambió alguien a vivir temporalmente en el hogar donde (NOMBRE) vivía para ayudarle?" Los informantes reportaron que, durante el último año de vida del adulto mayor fallecido, en 13.1 por ciento de los casos alguien se fue a vivir con él para apoyarlo en las actividades diarias. La segunda pregunta indaga si "En el último año de su vida, ¿Se cambió a vivir (NOMBRE) temporalmente con alguien?" Los resultados señalan que en 13.7 por ciento de los casos el fallecido se cambió a vivir a la casa de algún familiar. En ambos casos, los cambios en los arreglos residenciales involucran a los hijos y/o nietos principalmente.

\section{Uso de servicios de salud}

El último año de vida es uno de los periodos en los que se intensifica el uso de los servicios de salud, con amplias diferencias entre los individuos, donde algunos de ellos no utilizaran servicios o solo uno, mientras que otros harán uso de múltiples servicios. Desde una visión económica (costos), se ha documentado que a cualquier edad, el último año de vida es más costoso en términos de utilización de servicios de salud que cualquier año de vida anterior.

Al momento de fallecer 78.8 por ciento de los fallecidos tenía acceso a servicios de salud en alguna institución; 65.2 por ciento estuvo hospitalizado durante el último año de vida y en promedio estuvieron hospitalizados 17.4 noches. El lugar en el que regularmente recibían atención médica fue: Instituto Mexicano del Seguro Social (IMSS) con 51 por ciento, el Instituto de Seguridad y Servicios Sociales de los Trabajadores del Estado (ISSSTE) con 12.7 por ciento, la Secretaría de Salud (SSA) con 19.8 por ciento, y en instituciones privadas 16.5 por ciento. De los que durante el último año estuvieron hospitalizados 29.2 por ciento pagó por la hospitalización y el gasto promedio fue de 26,541 pesos. $^{2}$

\footnotetext{
${ }^{2}$ El Salario Mínimo Mensual en 2012 fue de \$1,870 pesos, y el tipo de cambio peso-dólar en ese mismo año fue de 13 pesos por dólar.
} 
Además de las hospitalizaciones, el número de visitas al médico es un indicador del uso de los servicios de salud y también es un predictor de mortalidad. Los informantes reportaron que 88.7 por ciento de los fallecidos visitó a un médico durante su último año de vida y que en promedio asistieron 13 veces. Complementando la información sobre uso de servicios de salud y los gastos asociados a ellos, la mitad (50.8 por ciento) tuvo gastos de bolsillo y en promedio se pagó $\$ 5,345$ pesos por gastos relacionados con la hospitalización, entre ellos transporte o medicamentos que no había o no cubría la institución. Cuando hubo gastos de bolsillo, por lo general los hijos, el cónyuge o la persona que falleció fueron los que pagaron por los servicios médicos (Tabla 1).

Debido a la importancia de los servicios de salud para las personas adultas mayores es necesario hacer un análisis más profundo sobre las diferencias en el uso de los servicios de salud según condición de derechohabiencia. ${ }^{3}$ La población que tenía derechohabiencia ( 78.8 por ciento de la población), en promedio pasó 5.5 noches más en un hospital que la población que no tenía derechohabiencia (18.2 contra 12.7 días). Una diferencia importante se encuentra en el porcentaje que pagó por hospitalización, mientras que entre los que tenían derechohabiencia en alguna institución de salud 21.9 por ciento pagó por hospitalización (quizás por tener los recursos económicos y preferencia por atenderse en otra institución) el porcentaje entre los que no tienen derechohabiencia fue de 81.8 por ciento. Entre los que pagaron por la hospitalización, para los que tenían derechohabiencia en promedio se pagó 29,100 pesos y entre los que no la tenían el monto fue de 21,427 pesos. Y finalmente en promedio los que tenían derechohabiencia fueron 13.7 veces al médico y entre los que no tenían el promedio fue 10.1 veces (Tabla 2).

Al hacer un análisis de hospitalización y enfermedades crónicas, los que fallecieron por enfermedad durante el último año, en promedio, pasaron 15.5 noches hospitalizados mientras que para los que fallecieron por accidente el promedio fue de 19.2 noches.

Para los que la principal causa de muerte fue cáncer el promedio de noches que estuvieron hospitalizados fue 17.6, para los de diabetes 15.5 noches, los de ataque al corazón 13.7 y para los de embolia el promedio de noches hospitalizado fue 10.7 .

\footnotetext{
${ }^{3}$ Según el Instituto Nacional de Estadística y Geografía, derechohabiencia se define como el "derecho de las personas a recibir servicios médicos en instituciones de salud públicas o privadas, como resultado de una prestación laboral por ser pensionado o jubilado, por inscribirse o adquirir un seguro médico o por ser familiar designado beneficiario".
} 


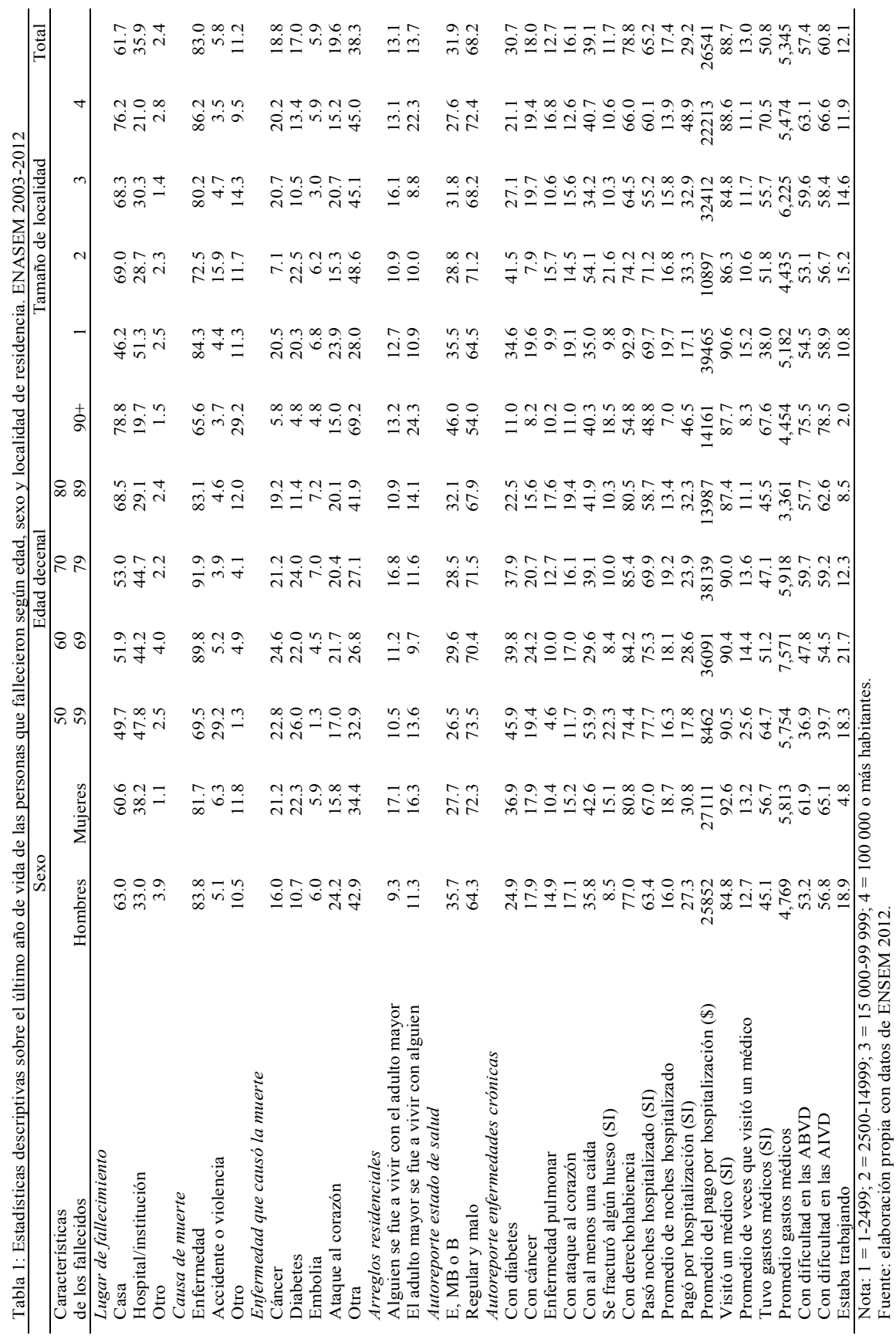


Tabla 2: Estadísticas descriptivas sobre hospitalización y servicio médico según derecho a servicio médico. ENASEM 2003.2012

\begin{tabular}{lrrr}
\hline & Sin derecho & Con derecho & Total \\
\hline Noches hospitalizado & 12.7 & 18.2 & 17.3 \\
Pagó por hospitalización & 81.8 & 21.9 & 29.4 \\
Cantidad por hospitalización & $\$ 21,428$ & $\$ 29,100$ & $\$ 26,421$ \\
Fue al médico & 78.9 & 91.4 & 88.7 \\
Veces al médico & 10.1 & 13.7 & 13.0 \\
\hline
\end{tabular}

Fuente: elaboración propia con datos de ENASEM 2012

Aprovechando la información con que cuenta el ENASEM, se presenta un análisis sobre las diferencias en el uso de servicios de salud entre la población viva y la fallecida para probar la hipótesis de que en el último año de vida se intensifica el uso de los servicios de salud. Los resultados señalan que mientras 65.2 por ciento de los que fallecieron se hospitalizaron, entre los vivos el porcentaje fue mucho menor con diez por ciento; también se encontró que los fallecidos pasaron más noches en el hospital, 17.4 con respecto a las 6.6 noches que pasaron los vivos (datos no mostrados). Con estas dos comparaciones básicas se comprueban las grandes diferencias que existen en el uso de los servicios de salud entre la población viva y la fallecida, y que en el último año de vida el uso de los servicios de salud se intensifica, tal y como sucede en otros contextos (Werblow, Felder y Zweifel, 2007).

\section{Discapacidad}

La discapacidad física puede ser congénita o ser resultado de enfermedad, lesión o deterioro físico. Un gran porcentaje de personas que viven hasta los 80 o 90 años están libres de discapacidad y son capaces de vivir independientemente, sin embargo, en la vejez existe una alta probabilidad de estar marcado por la discapacidad (Smith et al., 2013). Según lo reportado por los informantes del ENASEM, debido a problemas de salud, durante el último año de su vida 57.4 por ciento de los fallecidos recibieron ayuda con las Actividades Básicas de la Vida Diaria (ABVD: comer, bañarse, vestirse, caminar, acostarse y levantarse de la cama y usar el excusado). Los que generalmente les proporcionaban esa ayuda eran el cónyuge (15.7 por ciento), hijo/hija (63.3 por ciento) y nuera/yerno (6.7 por ciento). Con respecto a la intensidad con la que le brindaron cuidados, en 85 por ciento de los casos le ayudaban todos los días y en 40 por ciento de los casos la ayuda era las 24 horas del día, un indicador que nos acerca a la intensidad con la que recibían los cuidados es el promedio de horas de apoyo recibi- 
das, el cual fue de 14 horas al día. En cuanto a las diferencias por sexo, un porcentaje mayor de mujeres que de hombres tenían dificultad con las ABVD; por grupo de edad, los resultados señalan que 36.9 por ciento de los fallecidos que tenían entre 50 y 59 años tenían dificultad y el porcentaje se incrementa a 75.5 por ciento entre los de 90 años o más.

En cuanto a las Actividades Instrumentales de la Vida Diaria (AIVD: preparar una comida caliente, hacer compras de víveres/mandado, tomar sus medicamentos (si tomaba alguno) y manejar su dinero), 60.8 por ciento de los fallecidos recibieron ayuda en este tipo de actividades. Al igual que con las ABVD, generalmente la ayuda la recibieron del cónyuge (17.2 por ciento), hijo (60.2) y nuera/yerno (8.3 por ciento).

Los resultados sobre discapacidad y dependencia en el último año de vida coinciden con lo tradicionalmente reportado, el cuidado recae en los miembros de la familia, principalmente cónyuge e hijos, es prácticamente nula la presencia de cuidadores formales o que reciben una remuneración por el cuidado de la persona mayor, y la dependencia se acentúa en las edades muy avanzadas.

\section{Participación económica}

Además de las condiciones de salud y discapacidad, es importante conocer la situación económica de las personas adultas mayores durante su último año de vida. Contar con recursos monetarios y bienes es un elemento importante para sortear las vicisitudes que se presentan en las edades avanzadas, en la mayoría de las veces ayudan a enfrentar de mejor manera las problemáticas asociadas con la vejez. Entre los que fallecieron, según lo reportado por el informante, 12.1 por ciento estaban trabajando en el último año de vida, con amplias diferencias por género y grupo de edad (Tabla 1). De los que no trabajaban, 24.1 por ciento se dedicaba a los quehaceres del hogar, 29.3 por ciento no trabajaba por edad avanzada, 20.8 por ciento estaba retirado, 17.5 por ciento estaba enfermo o temporalmente discapacitado y 5.9 por ciento no podía trabajar para el resto de su vida.

En síntesis, las estadísticas descriptivas nos dejan ver un panorama complejo, heterogéneo y acorde a lo esperado, las mujeres con condiciones menos favorables que los hombres, los que vivían en localidades rurales también presentaban desventajas con respecto a su contraparte más urbana, y por grupo de edad las diferencias no son constantes ni van en un sentido de deterioro, quizás mediado por selectividad debido a mortalidad y en las edades muy avanzadas se observa a los más sanos o fuertes de una cohorte. Pareciera que después de alcanzar ciertas edades (80 o más) el estado 
de salud es bueno, influenciado por la percepción del estado de salud que deberían tener las personas —o al menos el que los informantes sustitutos consideran deberían tener las personas adultas mayores - una vez que alcanzan edades de 80 años o más.

\section{Factores asociados a noches de hospitalización}

Una de las principales preocupaciones del estudio sobre lo que pasa en el último año de vida y que de manera recurrente se presenta en la literatura es el uso intensivo de los servicios de salud y los factores asociados con ello, para profundizar en las condiciones de salud que las personas mayores tuvieron durante su último año de vida se estimó un modelo de regresión lineal sobre los factores asociados con el número de noches que pasó hospitalizada la persona adulta mayor. Los resultados señalan que contrario a lo que se esperaba, una mayor edad está asociada con menos noches de hospitalización, los que tenían derecho a servicios de salud en promedio pasaron más noches hospitalizados ( 4.7 noches) que aquellos que no tienen acceso; los que tuvieron dificultad para realizar las ABVD en promedio pasaron 5.6 noches más hospitalizados que los que no tenían dificultad, el número de noches que pasaron hospitalizados aquellos que tenían diagnosticado cáncer es mayor (3.9 noches), y los que por problemas de memoria no podían estar solos por más de una hora (3.2 noches).

En el modelo de regresión también se incluyeron variables sobre caídas, dificultad con las AIVD, diabetes, enfermedad pulmonar, ataque al corazón o embolia, y sexo pero ninguna tuvo una asociación significativa con la cantidad de noches que se pasó hospitalizado (Tabla 3).

\section{DisCUSIÓn}

Describir y analizar las condiciones de salud, los arreglos residenciales y el uso de servicios de salud que ocurren durante el último año de vida de las personas mayores que fallecieron entre 2003 y 2012 agrega nuevos elementos para entender el proceso de envejecimiento que se está presentando en México, así como las características y condiciones que presenta la población envejecida durante su último año de vida.

Estudiar el último año de vida de las personas mayores en México es importante, ésta investigación muestra una visión complementaria de lo que se ha venido realizando para explicar el proceso morbilidad-mortalidad de la población en edades avanzadas en México (García-González et al., 2009; González-González, Palloni y Wong, 2015). 


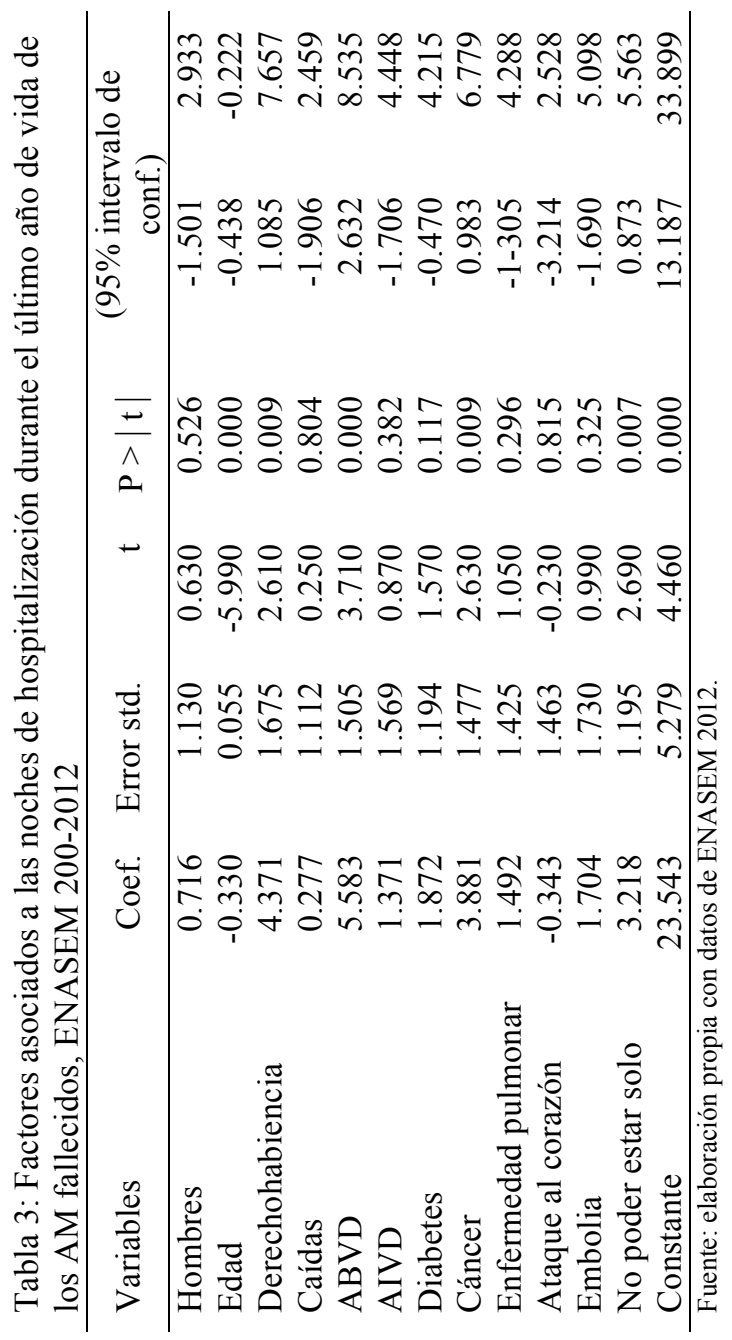


Contar con información longitudinal, como la del ENASEM, permite ampliar los horizontes de investigación, analizar la mortalidad y sus factores asociados o los factores predictores, estimar probabilidades de fallecer, así como analizar la evolución del estado de salud y explorar las condiciones de cómo fue el último año de vida y la posibilidad de poder relacionarlo con otros momentos en el tiempo (rondas previas del ENASEM).

Las principales causas de muerte entre la población envejecida fueron ataque al corazón, cáncer y diabetes. Particularmente con la diabetes, es necesario mencionar las amplias diferencias que se presentan en los niveles de mortalidad en los distintos tamaños de localidad de residencia. En las localidades urbanas, más que en las localidades rurales, fue más frecuente que la diabetes se reportara como la principal causa de muerte. La importancia y relevancia de estos hallazgos radica en el incremento de la prevalencia de diabetes entre la población de adultos (30-59 años), las consecuencias de la enfermedad y los estilos de vida que se presentan en los ámbitos urbanos, junto con resultados de proyecciones que señalan un futuro en el que la diabetes representará una carga mayor (Boyle et al., 2010).

La familia continua siendo uno de los principales pilares en el cuidado y apoyo hacia las personas mayores en México (Herrera y García, 2008), nuestros resultados reflejan cambios importantes en los arreglos residenciales para ayudar/apoyar a las personas en las actividades de la vida diaria durante su último año de vida. Sin embargo, es necesario buscar alternativas de cuidado ya que en el futuro la capacidad de apoyo familiar podría verse mermada debido a la menor cantidad de hijos que están teniendo las familias, los nuevos patrones de migración interna (hijos residiendo en un estado diferente al de los padres), las preferencias de las personas mayores por vivir solos y las nuevas dinámicas familiares que podrían reconfigurar los apoyos en términos de arreglos residenciales.

Nuestro interés por estudiar lo que sucede durante el último año de vida surge, principalmente, de la necesidad de comprobar la hipótesis de que en este periodo se hace un uso más intensivo de los servicios de salud. Al igual que en otros países (Hogan et al., 2001; Lynn, 2005), se encontró que los que fallecieron - con respecto a los que estaban vivos al momento de la entrevista - pasaron más tiempo hospitalizados, requirieron de más ayuda, tuvieron más gastos asociados con salud y realizaron más visitas al médico. Así pues, la vejez está relacionada con el uso intensivo de los servicios de salud, pero es el periodo de cercanía a la muerte en el que la demanda de esos servicios se incrementa sustancialmente. 
Para analizar la mortalidad y lo que sucede en el último año de vida de la población envejecida, es necesario reconocer que las condiciones sociales y familiares están cambiando, que son demasiados los factores involucrados en la forma en cómo se está envejeciendo, que algunos de esos factores actúan a favor y otros en contra de un mejor envejecimiento, lo que lleva a tener un grupo heterogéneo, con diferencias por edad, sexo y tamaño de localidad en que se reside. Lo cierto es que, durante el último año de vida, la presencia de enfermedades crónicas, dificultad en las actividades de la vida diaria, apoyo por parte de la familia y el uso de servicios de salud son una constante tanto para el individuo como para la familia, lo cual hace necesario realizar más investigación científica que permita generar acciones encaminadas a reducir las problemáticas que se presentan durante el último año de vida de las personas mayores.

\section{REFERENCIAS BIBLIOGRÁFICAS}

Alonso, P. et al., 2007, "Envejecimiento poblacional y fragilidad en el adulto mayor", en Revista Cubana de Salud Pública, 33(1), pp. 0-0. doi: 10.1590/S086434662007000100010 .

Boyd, C. M. et al., 2008, "Recovery in activities of dialy living among older adults followinng hospitalization for acute medical illness", en J Am Geriatr Soc., 56(12), pp. 2171-2179. doi: 10.1111/j.1532-5415.2008.02023.x.Recovery.

Boyle, J. P. et al., 2010, "Projection of the year 2050 burden of diabetes in the US adult population: Dynamic modeling of incidence, mortality, and prediabetes prevalence", en Population Health Metrics. BioMed Central Ltd, 8(1), p. 29. doi: 10.1186/1478-7954-8-29.

CONAPO, 2014, Dinámica demográfica 1990-2010 y proyecciones de población México. México.

Córdova-Villalobos, J. Á. et al., 2008, "Las enfermedades crónicas no transmisibles en México: sinopsis epidemiológica y prevención integral”, en Salud Pública de México, 50(5), pp. 419-427. doi: 10.1590/S0036-36342008000500015.

Coutinho, E. S. F., Bloch, K. V. y Coeli, C. M., 2012, “One-year mortality among elderly people after hospitalization due to fall-related fractures: comparison with a control group of matched elderly", en Cadernos de Saúde Pública, 28(4), pp. 801-805. doi: 10.1590/S0102-311X2012000400019.

Frankenberg, E. y Jones, N. R., 2004, "Self-rated health and mortality: Does the relationship extend to a low income setting?", en Journal of Health and Social Behavior, 45(4), pp. 441-452. doi: 10.1177/002214650404500406.

García-González, J. J. et al., 2009, “A frailty index to predict the mortality risk in a population of senior mexican adults", en BMC Geriatrics, 9(1), p. 47. doi: 10.1186/1471-2318-9-47. 
González-González, C. et al., 2014, "Mortality inequality among older adults in Mexico: the combined role of infectious and chronic disease", en Revista Panamericana de Salud Pública, 35(2), pp. 89-95. doi: 10.1016/j.biotechadv.2011.08.021. Secreted.

González-González, C., Palloni, A. y Wong, R., 2015, "Mortality and its association with chronic and infectious diseases in Mexico: a panel data analysis of the elderly”, en Salud Pública de México, 57(suppl. I), pp. S39-S45.

Herrera, E. and García, J., 2008, “El envejecimiento demográfico en México. Principales tendencias y características", en Horizontes, (13), pp. 93-100, recuperado de http://www.portal.conapo.gob.mx/publicaciones/sdm/sdm2008/06.pdf.

Hogan, C. et al., 2001, “'Medicare beneficiaries' costs of care in the last year of life”, en Health Affairs, 20(4), pp. 188-195. doi: 10.1377/hlthaff.20.4.188.

Hwang, I. et al., 2016, "Medical Costs and healthcare utilization among cancer decedents in the last year of life in 2009", en Cancer Research and Treatment, 48(1), pp. 365-375. doi: 10.4143/crt.2014.088.

Lubitz J.D. and Riley G.F., 1993, “Trends in medicare payments in the last year oflife", en Nejm, 328(15), pp. 1092-1096. doi: 10.1056/NEJM199304153281506.

Lunney, J. R. et al., 2003, "Patterns of functional decline at the end of life", en JAMA: the journal of the American Medical Association, 289(18), pp. 2387-2392. doi: 10.1001/jama.289.18.2387.

Lynn, J., 2005, Living long in fragile health: the new demographics shape end of life care, The Hastings Center report, Spec No (December), pp. S14-S18. doi: 10.1353/hcr.2005.0096.

Macías-Vega, D., 2004, "Arreglos residenciales de los adultos mayores", en La situación demográfica de México, 2004. México, Consejo Nacional de Población, pp. 43-52.

Marín, D. C. y Casanovas, G. L. I., 2001, Vejez, dependencia y cuidados de larga duración Situación actual y perspectivas de futuro, Colección Estudios Sociales. Edited by F. la "Caixa". Barcelona.

Mohan, V. et al., 2008, "Urban rural differences in prevalence of self-reported diabetes in India-The WHO-ICMR Indian NCD risk factor surveillance", en Diabetes Research and Clinical Practice, 80(1), pp. 159-168. doi: 10.1016/j.diabres.2007.11.018.

Monteverde, M. et al., 2010, "Obesity and excess mortality among the elderly in the United States and Mexico", en Demography, 47(1), pp. 79-96. doi: 10.1353/ dem.0.0085.

Muradás Troitiño, M., 2010, Las causas de muerte de los adultos mayores en México. Expresión de la desigualdad social, El Colegio de México, México.

Payne, G. et al., 2007, “Counting backward to health care's future: Using timeto-death modeling to identify changes in end-of-life morbidity and the impact of 
aging on health care expenditures", en Milbank Quarterly, 85(2), pp. 213-257. doi: 10.1111/j.1468-0009.2007.00485.x.

Prince, M. et al., 2012, "Dementia incidence and mortality in middle-income countries, and associations with indicators of cognitive reserve: A 10/66 Dementia Research Group population-based cohort study", en The Lancet. Elsevier Ltd, 380(9836), pp. 50-58. doi: 10.1016/S0140-6736(12)60399-7.

R. Todd Jewell, Maximo Rossi, P. T., 2007, "El estado de salud del adulto mayor en América Latina", en Cuadernos de Economía, vol. 26(46), pp. 147-167. Recuperado de http://www.scielo.org.co/scielo.php?script=sci_arttext\&pid=S0121477 $22007000100006 \& n r m=$ iso.

Schonberg, M. A. et al., 2009, "Index to predict 5-year mortality of communitydwelling adults aged 65 and older using data from the national health interview survey", en Journal of General Internal Medicine, 24(10), pp. 1115-1122. doi: 10.1007/s11606-009-1073-y.

Smith, A. K. et al., 2013, "Disability during the last two years of life", en JAMA Internal Medicine, 173(16), pp. 1506-1513. doi: 10.1001/jamainternmed.2013.8738.

Werblow, A., Felder, S. y Zweifel, P., 2007, "Population ageing and health care expenditure: A school of "Red Herrings"?", en Health Economics, 16(10), pp. 1109-1126. doi: 10.1002/hec.1213

\section{RESUMEN CURRICULAR DE LOS AUTORES}

\section{César González-González}

Maestro en Demografía por el Colegio de la Frontera Norte y Doctor en Estudios de Población por el Colegio de México. Ha sido investigador en el Instituto Nacional de Geriatría (2009-2016) y actualmente es Profesor Investigador de la Facultad de Trabajo Social en la Universidad de Colima. Es miembro del Sistema Nacional de Investigadores (Nivel 1). Entre sus publicaciones recientes destacan "Projecting diabetes prevalence among Mexicans aged 50 years and older: The Future Elderly Model-Mexico (FEM-Mexico)" (BMJ Open, 2017) "The effect of adult children living in the United States on the likelihood of cognitive impairment for older parents living in Mexico" (Ethnicity \& Health, 2018) y "Mortality inequality among older adults in Mexico: the combined role of infectious and chronic diseases" (Rev Panam Salud Publica, 2014).

Dirección electrónica: cgonzalez31@ucol.mx 


\section{Karina Orozco Rocha}

Es Doctora en Estudios de Población por El Colegio de México. Maestra en Ciencias, área Finanzas por la Universidad de Colima. Sus líneas de investigación y publicaciones examinan las desigualdades de género en el trabajo remunerado y no remunerado. En la actualidad realiza un posdoctorado en la Universidad de Colima y estudia el bienestar económico y las redes de protección en las edades avanzadas de los mexicanos. Sus publicaciones más recientes son "Dynamics of Economic Security Among the Aging in Mexico: 2001-2012" (Population Research and Policy Review, 2018) y "Atrición en encuestas de panel en México: la Encuesta Nacional sobre Salud y Envejecimiento en México" (Realidad, Datos y Espacio, 2018).

Dirección electrónica: k_orozco@hotmail.com

\section{Mireya Patricia Arias Soto}

Licenciada en Trabajo Social, Maestra en Trabajo Social con Orientación en Desarrollo Humano y Familia por la Universidad de Colima, y Doctora en Educación Ph D., por la Universidad de Baja California. Profesora e Investigadora de Tiempo Completo de la Facultad de Trabajo Social de la Universidad de Colima, con Perfil PRODEP. Ponente en eventos académicos de investigación a nivel nacional e internacional de temas relacionados con: Familia, Adultos Mayores, Grupos Vulnerables, Migración, entre otros. Coautora de artículos de revista, capítulos de libros y libros de temas relacionados con Familia y Grupos Sociales Emergentes. Integrante del Cuerpo Académico (CA) UCOL-CA77 "Grupos Sociales y Trabajo Social", el cual se encuentra en Consolidación; a partir de enero 2014, Líder del CA. Integrante de la Academia Nacional de Investigación en Trabajo Social (ACANITS).

Dirección electrónica: mireya_arias@ucol.mx

\section{María Gregoria Carvajal Santillán}

Doctora en Gerencia y política educativa, Profesora e Investigadora de Tiempo Completo de la Facultad de Trabajo Social de la Universidad de Colima, Diplomada en Atención a la Vejez en Tiempos de Globalización, ha participado en los siguientes cursos y talleres: Narrativa experiencial: El curso y sentido de vida, resignificado en la tercera edad, La salud del adulto mayor, Alimentación básica para adultos mayores, Ave Fenix: Modelo de atención al adulto mayor, Cuidados básicos del adulto mayor, Reflexología para el adulto mayor, creatividad en el adulto mayor, Recreación y activa- 
ción física en el adulto mayor, ha colaborado en los siguientes proyectos de investigación: Envejecimiento y Mortalidad: Condiciones de salud, económicas y familiares en el último año de vida, Situación familiar del adulto mayor institucionalizado en el Municipio de Colima, Colima (2016), Diagnóstico de los adultos mayores institucionalizados en el Estado de Colima, Problemática por las que atraviesa el Adulto Mayor, Conciencia a Plenitud. Dirección electrónica: margre@ucol.mx

\section{Rafael Samper Ternent}

Médico Geriatra de la Pontificia Universidad Javeriana en Bogotá, Colombia y Doctor en Ciencia Clínicas de The University of Texas Medical Branch (UTMB) en Galveston, Estados Unidos. Es profesor Asistente en el Departamento de Medicina Interna de UTMB e Investigador del Sealy Center on Aging de la misma Universidad desde 2017. Desde 2009 es CoInvestigador de la Encuesta Nacional de Salud y Envejecimiento en México (ENASEM). Entre sus publicaciones recientes se destacan "Early Life Displacement Due to Armed Conflict and Violence, Early Nutrition, and Older Adult Hypertension, Diabetes, and Obesity in the Middle-Income Country of Colombia." (J Aging Health, 2018), "Patterns of SES health disparities among older adults in three upper middle- and two high-income countries." (J Gerontol B Psychol Sci Soc Sci, 2018) "Frailty and sarcopenia in Bogotá: results from the SABE Bogotá Study." (Aging Clin Exp Res, 2017).

Dirección electrónica: rasamper@utmb.edu

Artículo recibido el 9 de agosto de 2017 y aprobado el 25 de abril de 2018 . 